\title{
Electronic and Magnetic Properties of Rare-Earth Metals Doped ZnO Monolayer
}

\author{
Changlong Tan, ${ }^{1}$ Dianshuang Xu, ${ }^{1}$ Kun Zhang, ${ }^{1}$ Xiaohua Tian, ${ }^{1}$ and Wei Cai ${ }^{2}$ \\ ${ }^{1}$ College of Applied Science, Harbin University of Science and Technology, Harbin 150080, China \\ ${ }^{2}$ School of Materials Science and Engineering, Harbin Institute of Technology, Harbin 150001, China \\ Correspondence should be addressed to Changlong Tan; changlongtan@hrbust.edu.cn
}

Received 24 March 2015; Revised 29 June 2015; Accepted 2 July 2015

Academic Editor: Chaochao Dun

Copyright (c) 2015 Changlong Tan et al. This is an open access article distributed under the Creative Commons Attribution License, which permits unrestricted use, distribution, and reproduction in any medium, provided the original work is properly cited.

\begin{abstract}
The structural, electronic, and magnetic properties of rare-earth metals doped $\mathrm{ZnO}$ monolayer have been investigated using the first-principles calculations. The induced spin polarization is confirmed for $\mathrm{Ce}, \mathrm{Eu}, \mathrm{Gd}$, and Dy dopings while the induced spin polarization is negligible for $\mathrm{Y}$ doping. The localized $f$ states of rare-earth atoms respond to the introduction of a magnetic moment. $\mathrm{ZnO}$ monolayer undergoes transition from semiconductor to metal in the presence of $\mathrm{Y}, \mathrm{Ce}, \mathrm{Gd}$, and Dy doping. More interestingly, Eu doped $\mathrm{ZnO}$ monolayer exhibits half-metallic behavior. Our result demonstrates that the RE-doping is an efficient route to modify the magnetic and electronic properties in $\mathrm{ZnO}$ monolayer.
\end{abstract}

\section{Introduction}

$\mathrm{ZnO}$ is a wide band gap II-VI semiconductor which has several favorable properties, such as wide band gap, good transparency, and large exciton binding energy. It has been used for solar cells, light emitting devices, and transparent electrodes [1-9]. Recently, the interest in $\mathrm{ZnO}$ nanostructures has significantly increased owing to their specific structures and properties differ from bulk counterparts, leading to many potential applications. Several $\mathrm{ZnO}$ nanostructures have been synthesized and characterized [10-18], in particular in the form of ultrathin nanosheets. The two-dimensional layered phase of $\mathrm{ZnO}$ was firstly predicted by Freeman that $\mathrm{ZnO}$ film prefers a graphitic-like structure when the number of $\mathrm{ZnO}(0001)$ layers is reduced due to the depolarization of the surface $[19,20]$. Tusche et al. were the first to synthesize twomonolayer-thick $\mathrm{ZnO}(0001)$ films deposited on a $\mathrm{Ag}(111)$ surface, where $\mathrm{Zn}$ and $\mathrm{O}$ atoms are arranged in planar sheet like in the hexagonal BN monolayer [21]. Furthermore, graphenelike honeycomb structures of $\mathrm{ZnO}$ have been successfully prepared on $\operatorname{Pd}(111)$ substrate [22].

In order to design $\mathrm{ZnO}$-based devices, one of the most relevant issues is doping in pure $\mathrm{ZnO}$. Extensive studies have been conducted on the electronic and magnetic properties of the $\mathrm{ZnO}$ monolayer doped with foreign atoms for nanoelectronic and spintronic applications [23-27]. So far, it is well know that, by doping with nonmetal (B, C, and N) species in the graphene-like $\mathrm{ZnO}$ monolayer, or adsorptions of an $\mathrm{Mn}$ atom on a $\mathrm{ZnO}$ sheet, the tunable electronic and magnetic properties and ferromagnetic coupling can be realized. Very recently, a transition-metal-doped twodimensional $\mathrm{ZnO}$ monolayer has been investigated by firstprinciples calculations [26]. The results show that electronic and magnetic properties of $\mathrm{ZnO}$ monolayer can be modified by such doping. On the other hand, compared with $3 d$ transition metals, if rare-earth (RE) metals have larger magnetic moments. Furthermore, the electrons may mediate the FM coupling between the RE ions due to the coupling between $f$ electrons and host $s$ electrons. So far, although the doping of $\mathrm{RE}$ atom in $\mathrm{ZnO}$ bulk has been studied previously [28-32], the electronic and magnetic properties of RE-doped $\mathrm{ZnO}$ monolayer remain unclear. Thus, it is important to understand the electronic structure and magnetic properties of RE-doped $\mathrm{ZnO}$ monolayer due to its potential application in nanoelectronic and spintronic devices.

In this study, the structural, electronic, and magnetic properties of the RE-doped (RE $=\mathrm{Y}, \mathrm{Ce}, \mathrm{Eu}, \mathrm{Gd}$, and Dy) $\mathrm{ZnO}$ monolayer with $\mathrm{Zn}$ atoms substituted by $\mathrm{RE}$ atoms have been 


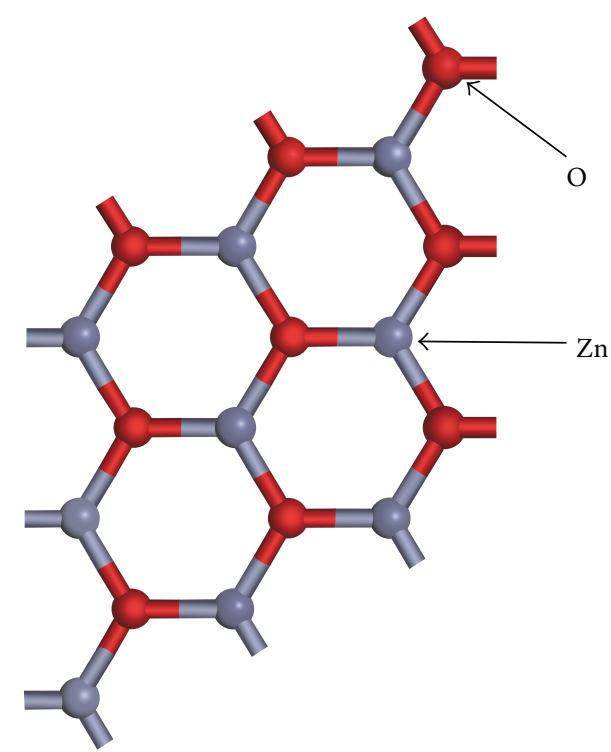

(a)

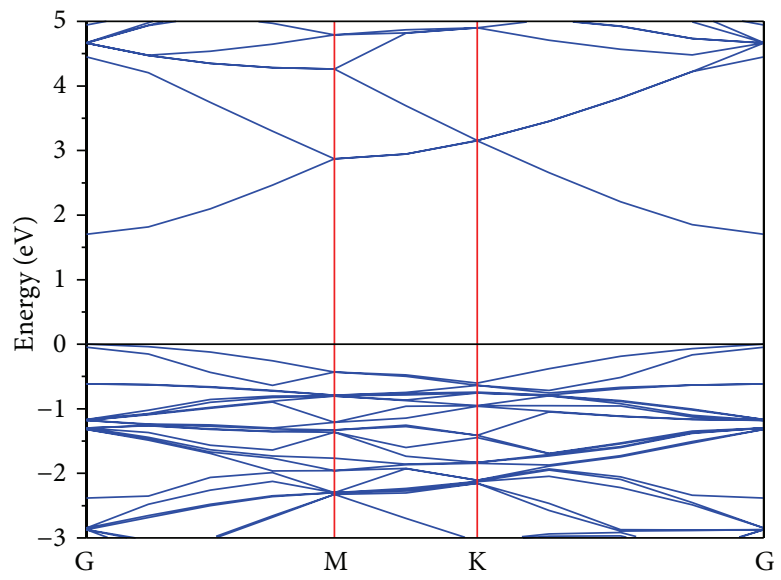

(b)

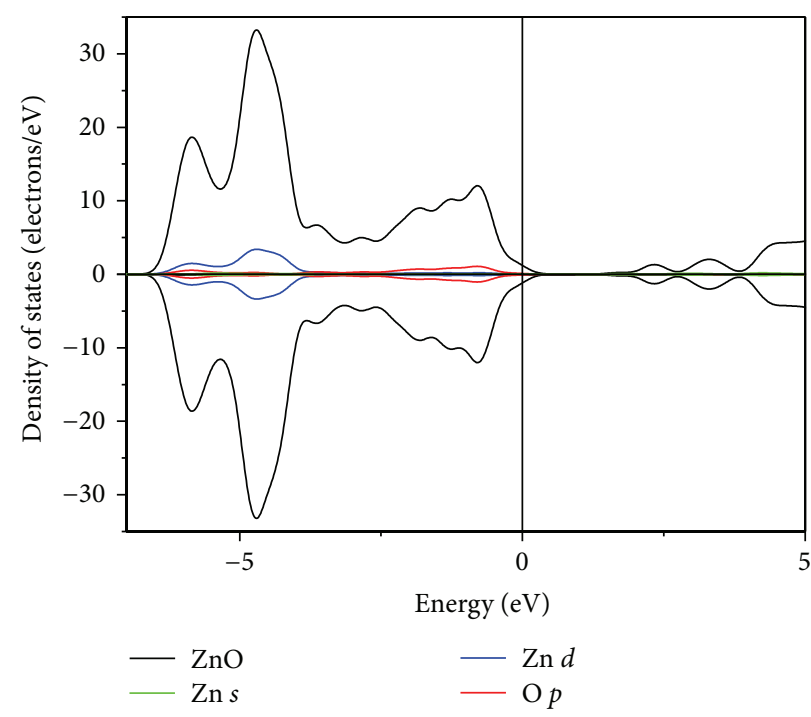

(c)

FIgURE 1: (a) Relaxed structure, (b) band structure, and (c) DOS of $\mathrm{ZnO}$ monolayer. The Fermi level is set to zero.

systematically studied by using first-principles calculations. It is found that the RE doping is an efficient route to tune the magnetic and electronic properties in $\mathrm{ZnO}$ monolayer and may provide a reference for its nanoelectronic and spintronic applications.

\section{Computational Methods}

First-principles calculations are performed within the framework of density functional theory (DFT) using the $\mathrm{DMol}^{3}$ package $[33,34]$. We used the generalized gradient approximation (GGA) with Perdew-Burke-Ernzerhof (PBE) functional to explain the exchange and correlation terms [35]. The geometry optimization is carried out with all atoms free to move and full cell optimization. The convergence criteria are of $1.0 \times 10^{-5}$ Hartree in energy, 0.002 Hartree/ $\AA$ in maximum force, and $0.005 \AA$ in maximum displacement. We set the orbital cutoff globally with a parameter of $4.2 \AA$, and smearing is 0.035 Hartree. The brillouin zone is sampled with $4 \times$ $4 \times 1$ k-points. The models of $3 \times 3$ supercells for $\mathrm{ZnO}$ monolayer with one $\mathrm{Zn}$ atom substituted by one $\mathrm{RE}$ ( $\mathrm{RE}=$ $\mathrm{Y}, \mathrm{Ce}, \mathrm{Eu}, \mathrm{Gd}$, and $\mathrm{Dy}$ ) atom are considered. The vacuum region of $15 \AA$ along the nonperiodic directions is employed to avoid interactions between two neighboring layers. All the calculations are carried out with spin polarization.

\section{Results and Discussion}

To investigate the properties of RE-doped $\mathrm{ZnO}$ monolayer, it is worth to mention the structural and electronic properties 
of the pristine $\mathrm{ZnO}$ monolayer. The initial structure of the $\mathrm{ZnO}$ monolayer is cleaved from a bulk wurtzite $\mathrm{ZnO}$ with (0001) polar surface. After full optimization, the pristine $\mathrm{ZnO}$ monolayer transforms from initial wurtzite structure with a rippled surface into a graphene-like plane structure, as shown in Figure 1(a). The relaxed bond length of $\mathrm{Zn}-\mathrm{O}$ in $\mathrm{ZnO}$ monolayer is $1.91 \AA$, which is shorter than its wurtzite bulk value of $2.01 \AA$. The contraction of the $\mathrm{Zn}-\mathrm{O}$ bond length is attributable to the fact that the $s p^{2}$ hybridization in twodimensional honeycomb structure is stronger than the $s p^{3}$ hybridization in wurtzite crystal. The geometric structure of the pristine $\mathrm{ZnO}$ monolayer agrees well with previous experimental and theoretical values $[20,21,23,36]$. The band structure and density of states (DOS) of the pristine $\mathrm{ZnO}$ monolayer are calculated after structural optimization and present in Figures 1(b) and 1(c), respectively. The calculated band structure indicates that pristine $\mathrm{ZnO}$ monolayer is a semiconductor with direct band gap of $1.70 \mathrm{eV}$, consistent with previous theoretical calculations [21, 23]. The spinup and spin-down components of the DOS are totally symmetric, indicating that the pristine $\mathrm{ZnO}$ monolayer is nonmagnetic. Moreover, it is found that the valence bands are dominated by $\mathrm{O} 2 p$ and $\mathrm{Zn} 3 d$ states, whereas the conduction bands are mainly ascribed to the $\mathrm{O} 2 p$ and $\mathrm{Zn} 4 s$ states.

In the following, considering spin polarization, we optimized the structures of RE-doped $\mathrm{ZnO}$ monolayer. The average bond lengths for the $\mathrm{Y}, \mathrm{Ce}, \mathrm{Eu}, \mathrm{Gd}$, and $\mathrm{Dy}$ atoms to their nearest-neighbor $\mathrm{O}$ atoms are 2.10, 2.19, 2.18, 2.14, and $2.16 \AA$, respectively. From the bond lengths, it can be seen that the RE-O bond is expanded a little compared to the $\mathrm{Zn}-\mathrm{O}$ of the pristine $\mathrm{ZnO}$ monolayer. The RE-O-Zn bond angles for the $\mathrm{Y}, \mathrm{Ce}, \mathrm{Eu}, \mathrm{Gd}$, and Dy atoms are 117.03, 115.06, $113.79,115.87$, and $115.20^{\circ}$, respectively. Considering the $\mathrm{Zn}$ $\mathrm{O}$ - $\mathrm{Zn}$ bond angle is $120^{\circ}$ in the pristine $\mathrm{ZnO}$ monolayer, it can be known that RE doping distorts the bond angle. From these results, we can find that compared to the pristine one, the RE-doped $\mathrm{ZnO}$ systems are distorted. The main reason is the different atomic radius between doping RE atoms and $\mathrm{Zn}$ atom.

The formation energy of the RE-doped $\mathrm{ZnO}$ monolayer has been calculated for the understanding of its relative stability. The definition of formation energy is given as $E_{f}=$ $E_{\text {tot }}(\mathrm{RE}$-doped $\mathrm{ZnO})-E_{\text {tot }}(\mathrm{ZnO})+\mu_{\mathrm{Zn}}-\mu_{\mathrm{RE}}$, where $E_{\text {tot }}$ (RE-doped $\mathrm{ZnO})$ and $E_{\text {tot }}(\mathrm{ZnO})$ are the total energies per supercell of the relaxed RE-doped and pure $\mathrm{ZnO}$ monolayer, respectively. The $\mu_{\mathrm{Zn}}$ and $\mu_{\mathrm{RE}}$ represent the chemical potential of $\mathrm{Zn}$ and RE species, respectively [37]. Figure 2 presents the results of calculated formation energies of the RE-doped $\mathrm{ZnO}$ monolayer. As displayed in the figure, the formation energies of all the doped systems are found to be negative. The smaller the formation enthalpy is, the easier the dopant incorporates into the $\mathrm{ZnO}$ sheet. The obtained results of the formation energies indicate that the RE atoms of $\mathrm{Y}, \mathrm{Ce}, \mathrm{Eu}, \mathrm{Gd}$, and Dy are suitable to dope into $\mathrm{ZnO}$ monolayer. This is also demonstrated by the experiment. Being directly related to the present work, successful Eu doping in $\mathrm{ZnO}$ nanowires has been experimentally achieved by ion implantation.

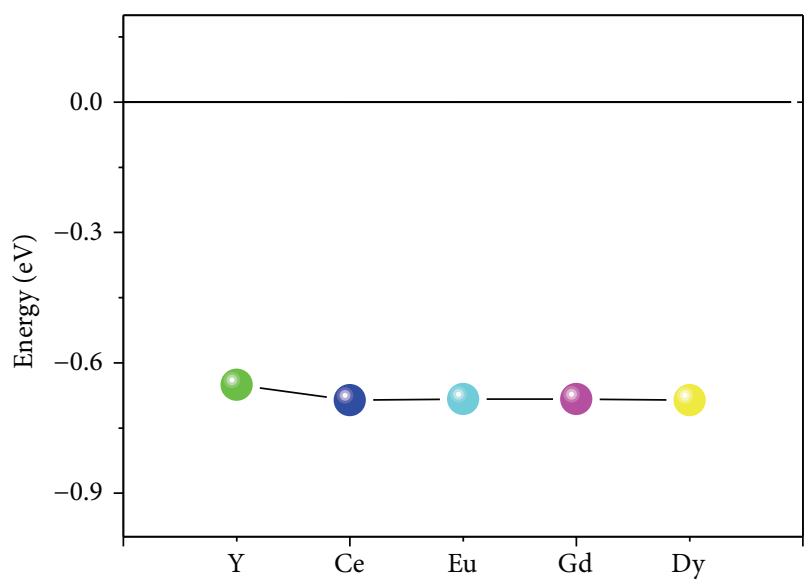

FIGURE 2: The formation energies of the RE-doped $\mathrm{ZnO}$ monolayer.

The incorporated $\mathrm{RE}$ atoms were found to replace $\mathrm{Zn}$ in the $\mathrm{ZnO}$ lattice [13].

An important aspect of RE-doped $\mathrm{ZnO}$ monolayer is the magnetic behavior of the system. From our calculation, it is found that no magnetism is observed when $\mathrm{Y}$ atom is doped in the $\mathrm{ZnO}$ monolayer. In the case of $\mathrm{Ce}, \mathrm{Eu}, \mathrm{Gd}$, and $\mathrm{Dy}$ doped system, the induced spin polarization is observed. The total magnetic moments and local magnetic moments of RE, $\mathrm{Zn}$, and $\mathrm{O}$ atoms in the considered systems are plotted with respect to a series of RE atoms as shown in Figure 3. The total magnetic moments of $\mathrm{ZnO}$ monolayer doped by $\mathrm{Ce}, \mathrm{Eu}, \mathrm{Gd}$, and Dy are $-0.76,3.43,3.78$, and $2.28 \mu_{\mathrm{B}}$, respectively. In the above case, the magnetic properties of the systems are mainly attributed to the contribution of doped RE impurities because of the nonmagnetic character of pristine $\mathrm{ZnO}$ monolayer. Moreover, one can see from Figure 3 that RE atoms have major contributions to the total magnetic moment and the nearest-neighbor $\mathrm{O}$ atoms only have very minor contribution to the total magnetic moment. This phenomenon is similar to the cases of $3 d \mathrm{TM}$-doped $\mathrm{ZnO}$ sheet and $\mathrm{BN}$ sheet [38], which was also observed in $3 d \mathrm{TM}$-doped $\mathrm{ZnO}$ nanotubes [39].

The magnetism distributions of RE-doped $\mathrm{ZnO}$ monolayer can be studied by the analysis of the spin density as shown in Figure 4. It can be seen that $\mathrm{Ce}, \mathrm{Eu}, \mathrm{Gd}$, and Dy doped $\mathrm{ZnO}$ monolayers exhibit similar distribution phenomenon that is magnetic moments mainly concentrated on the $\mathrm{RE}$ atoms and nearest-neighbor $\mathrm{O}$ atoms contributed slightly. This is consistent with above calculated local magnetic moments of RE and $\mathrm{O}$ atoms.

In order to further investigate the effects of RE doping and the origins of the magnetic properties, the spin polarized band structures and projected density of states of RE-doped $\mathrm{ZnO}$ monolayer have been calculated. The spin polarized band structures have been presented in Figure 5. From Figure 5(b), it can be seen that the majority and minority band structures of $\mathrm{ZnO}$ monolayer doped by $\mathrm{Y}$ are identical with zero magnetic moment of $Y$ atoms. Moreover, the system of $\mathrm{Y}$ doped $\mathrm{ZnO}$ monolayer is nonmagnetic metallic, which is well consistent with highly conductive films of $\mathrm{Y}$ doped 

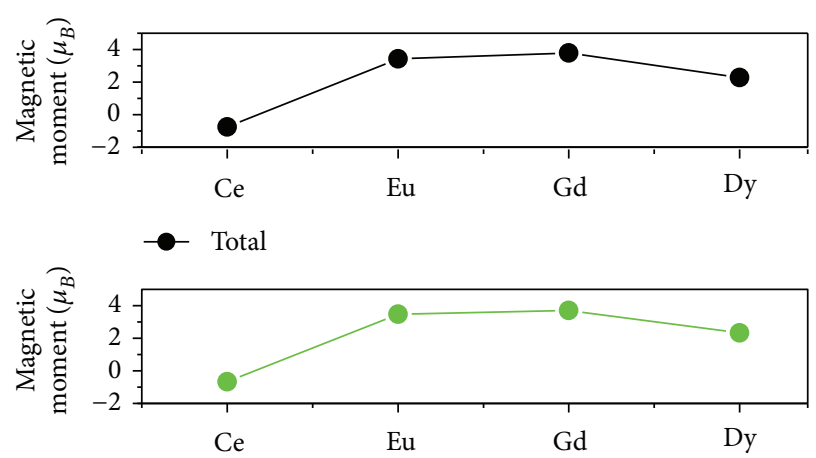

- RE
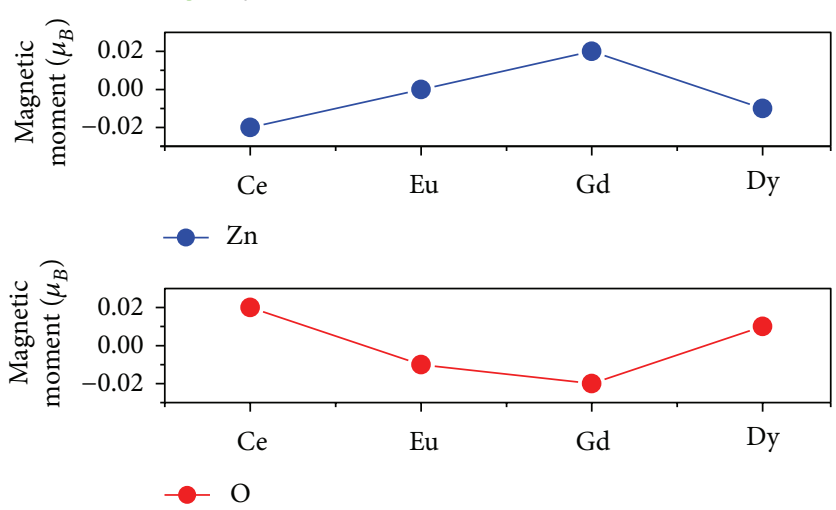

FIgUre 3: The total and local magnetic moments of RE, $\mathrm{Zn}$, and $\mathrm{O}$ atoms in the RE-doped $\mathrm{ZnO}$ monolayer.

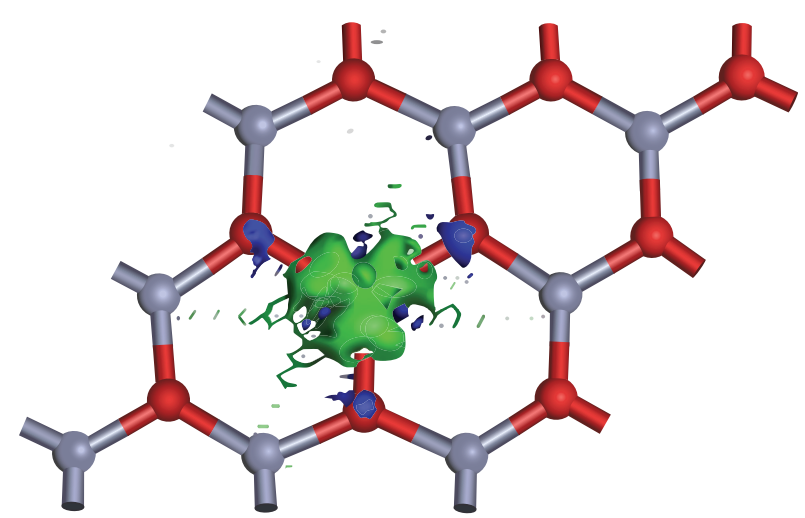

(a)

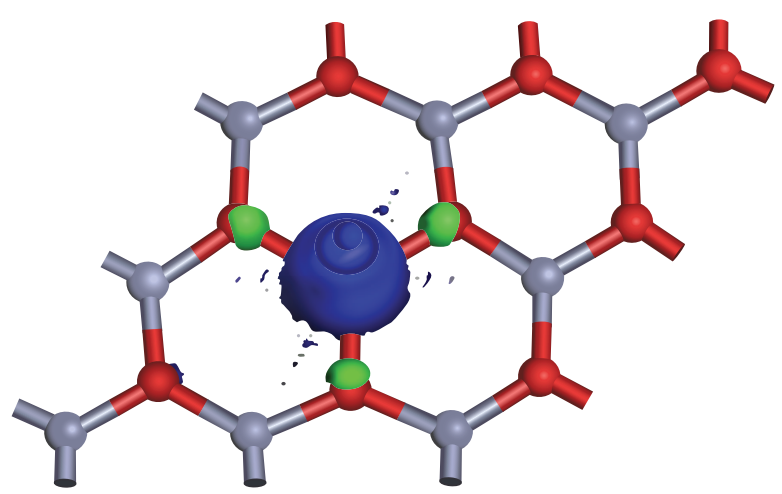

(c)

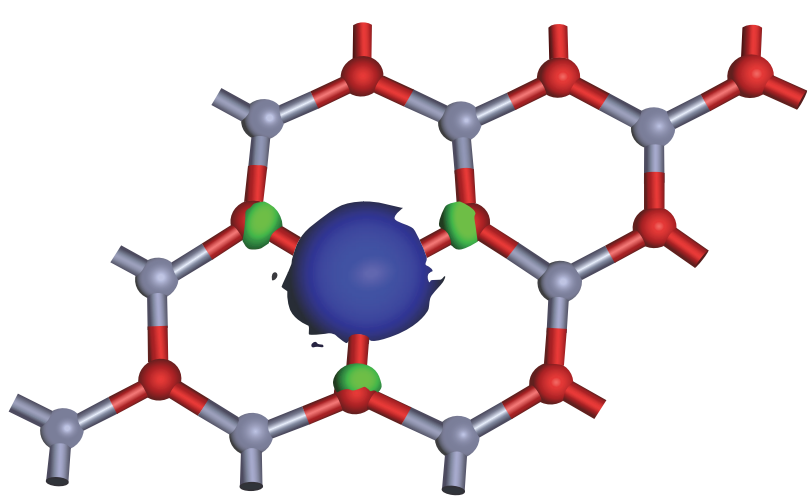

(b)

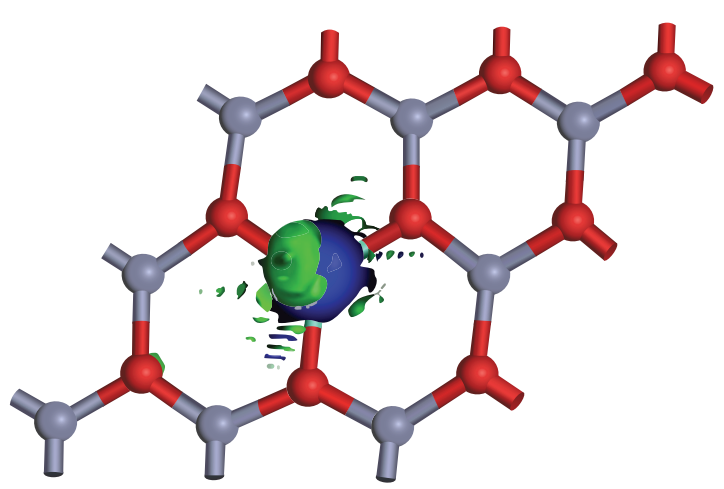

(d)

Figure 4: The spin charge density distribution of (a) Ce, (b) Eu, (c) Gd, and (d) Dy doped ZnO monolayers. The blue and green colors represent spin-up and spin-down values, respectively. 


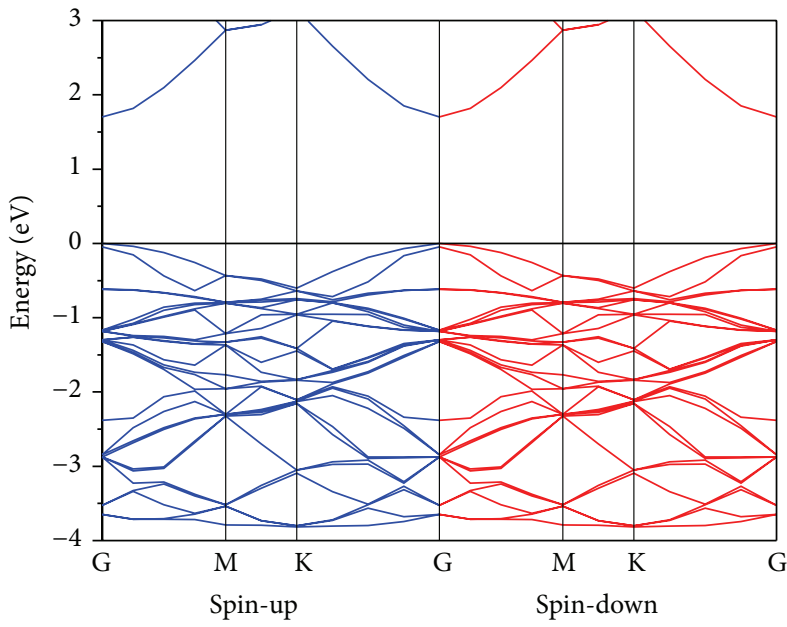

(a)

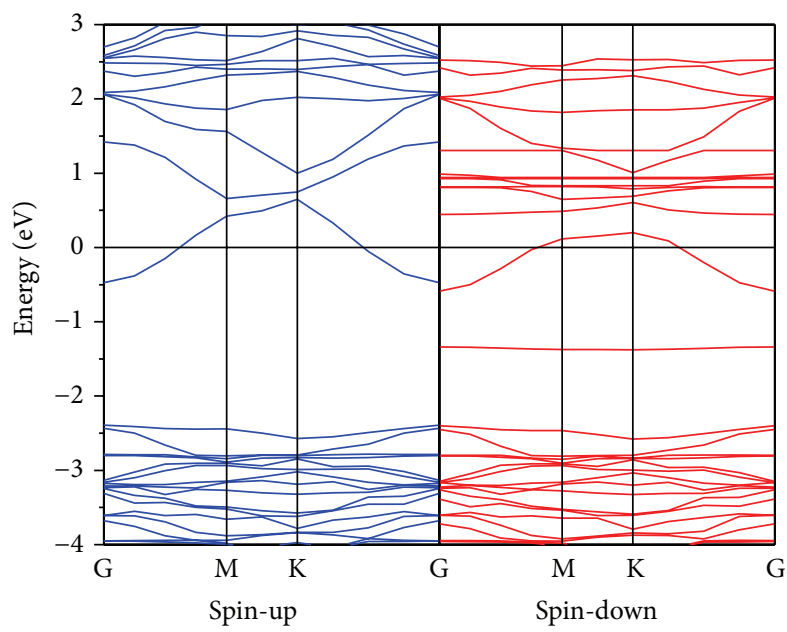

(c)

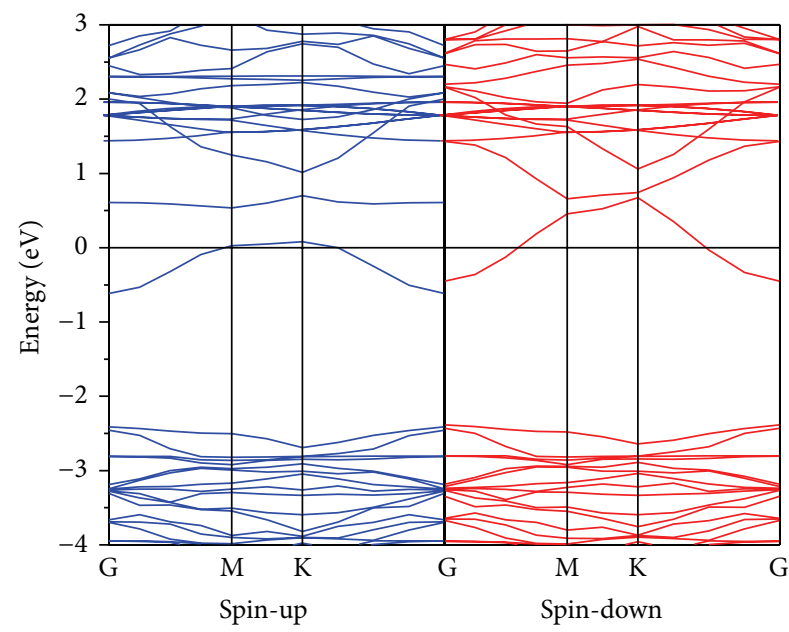

(e)

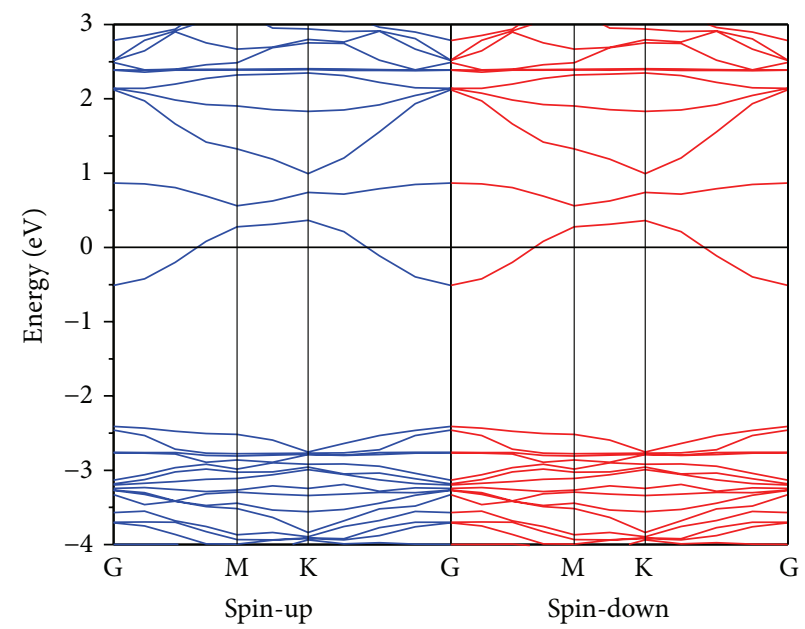

(b)

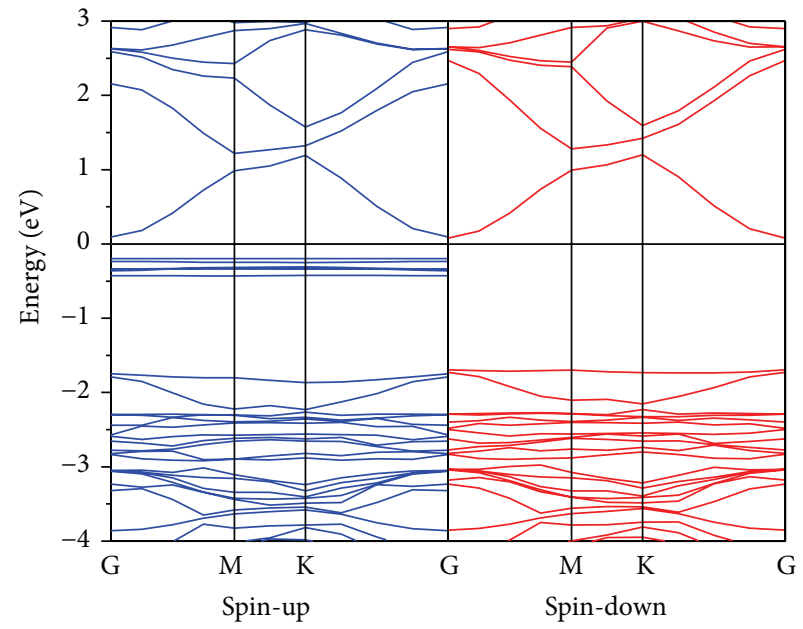

(d)

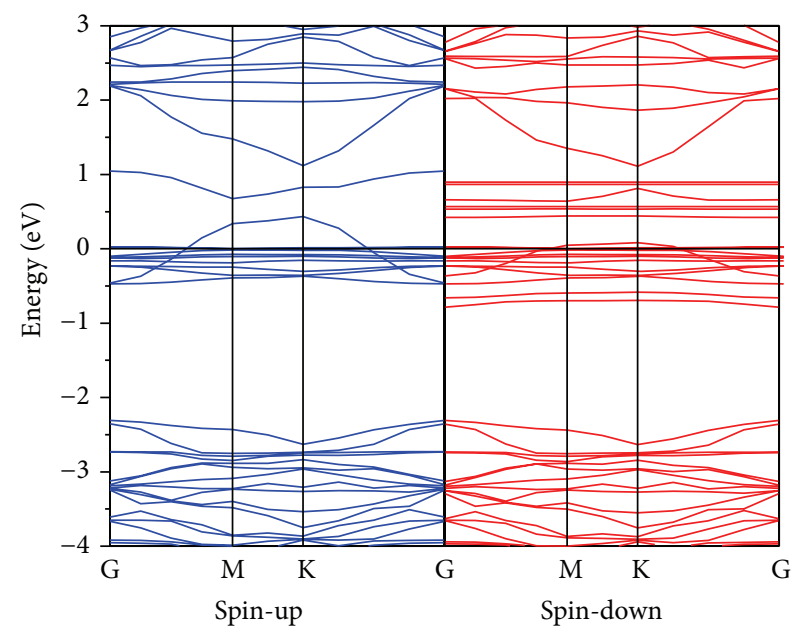

(f)

FIGURE 5: The spin polarized band structures of (a) pristine and (b)-(f) a single Y, Ce, Eu, Gd, and Dy doped ZnO monolayers. The horizontal dash line indicates the Fermi level. 

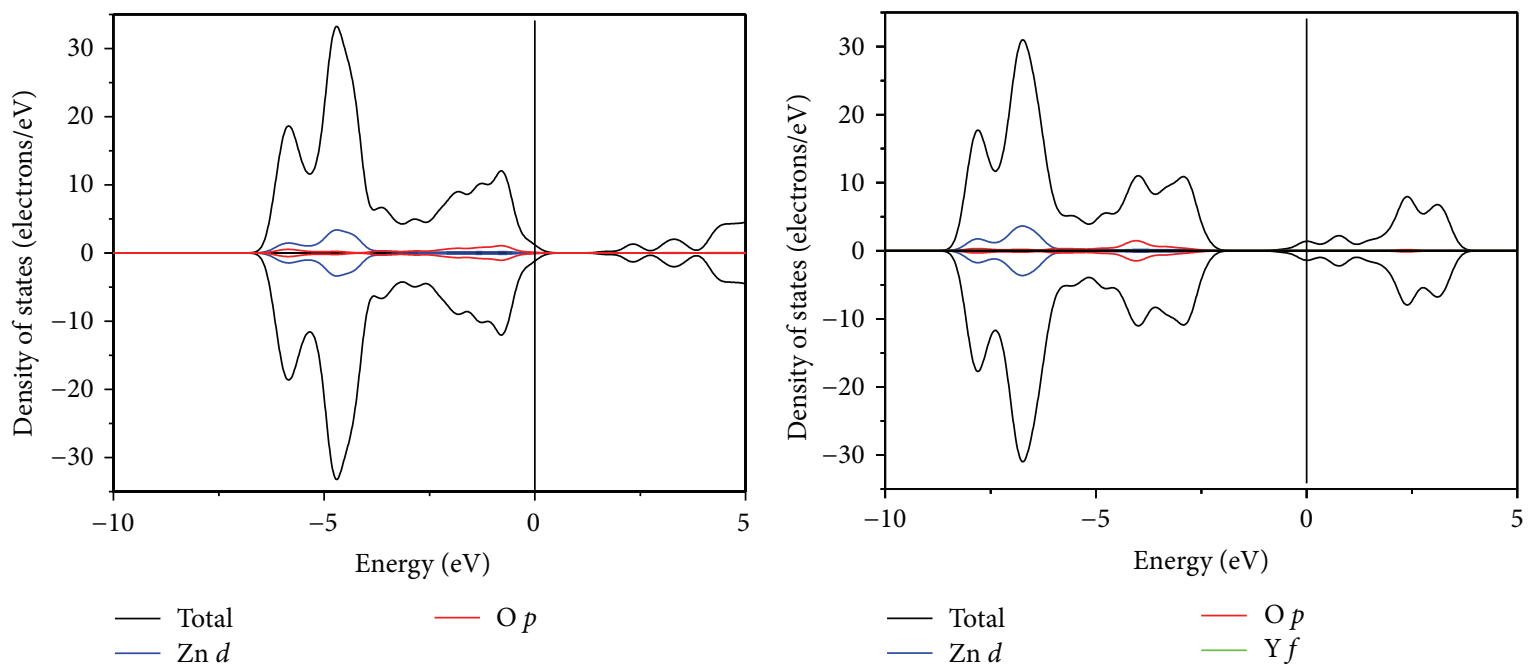

(a)

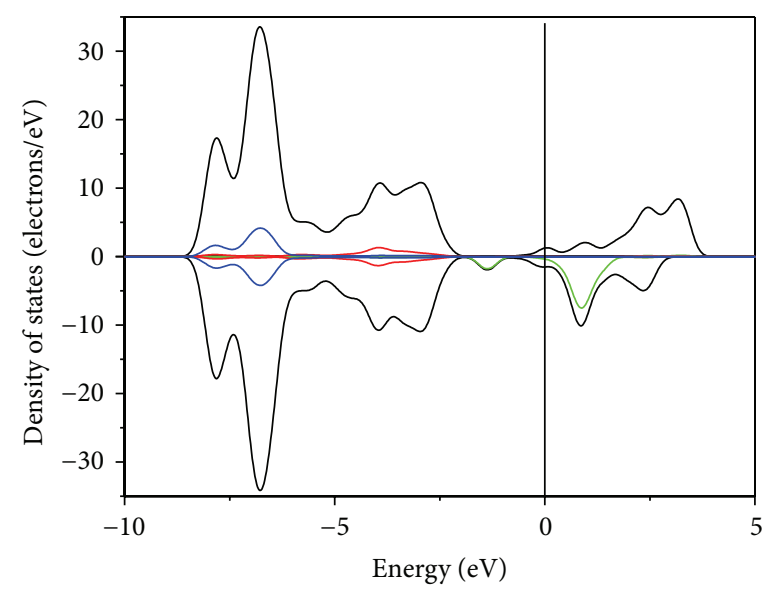

(b)

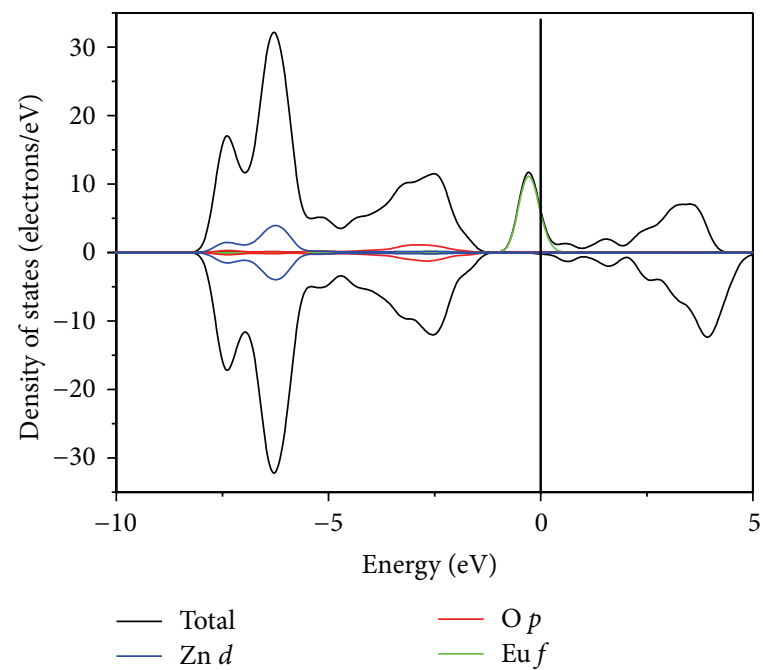

(c)

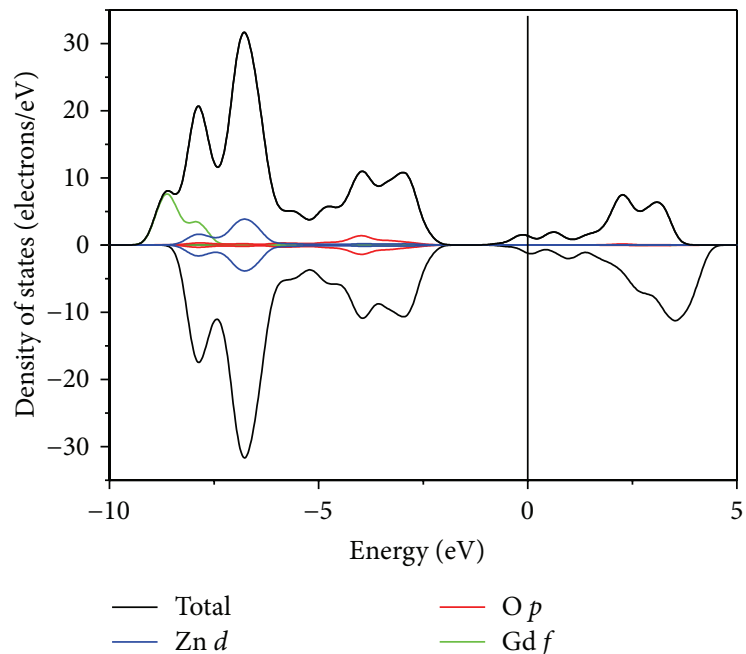

(e)

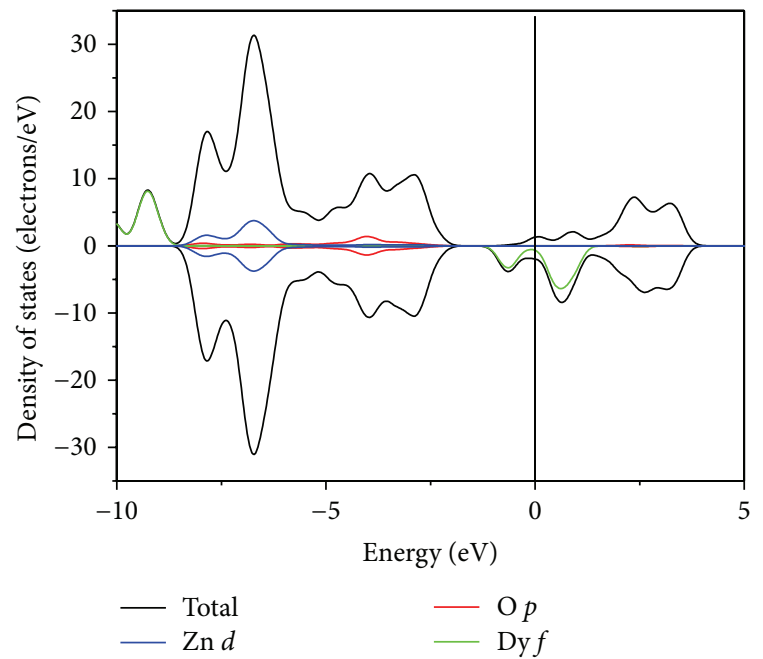

(f)

Figure 6: The total and partial DOS of (a) pristine and (b)-(f) a single Y, Ce, Eu, Gd, and Dy doped ZnO monolayers. The vertical dash line indicates the Fermi level. 
$\mathrm{ZnO}$ reported by Minami et al. [40]. In the case of $\mathrm{ZnO}$ monolayer doped by $\mathrm{Ce}, \mathrm{Gd}$, and $\mathrm{Dy}$, the calculated band structure as shown in Figures 5(c), 5(e), and 5(f) indicates that these systems are magnetic metallic with spin polarized bands cross the Fermi level for both spin-up and spin-down channels. Meanwhile, the majority bands in the vicinity of the Fermi level are different from the minority bands in which several nearly flat bands appear near $1.0 \mathrm{eV}$ below the Fermi level. The band structure of Eu doped $\mathrm{ZnO}$ monolayer is shown in Figure 5(d). It is worth noting that the spindown channel is semiconducting with a direct band gap of $1.8 \mathrm{eV}$, whereas it is important that the spin-up channel is metallic with impurity bands induced by Eu dopant crossing the Fermi level. As a result, the one Eu doped $\mathrm{ZnO}$ monolayer is magnetic half-metallic. The half-metallic nature with a $100 \%$ spin polarization at the Fermi level is considered as an optimal candidate for spintronic devices.

Figure 6 shows the total and partial DOS of RE-doped $\mathrm{ZnO}$ monolayer. As shown in Figure 6(b), for the Y doped $\mathrm{ZnO}$ monolayer, it can be seen that the spin-up and spindown DOSs are completely symmetrical, indicating the nonmagnetic states of the system. And it can also be seen that the $\mathrm{Ce}, \mathrm{Eu}, \mathrm{Gd}$, and Dy doped $\mathrm{ZnO}$ monolayers are magnetic because there is a clear spin polarization between the DOSs of the two spin channels near the Fermi level. The analyses of the total DOSs are consistent with those of calculated magnetic properties. Furthermore, for the $\mathrm{ZnO}$ monolayer doped by $\mathrm{Ce}, \mathrm{Eu}, \mathrm{Gd}$, and $\mathrm{Dy}$, partial DOS indicates that $f$ electrons of $\mathrm{RE}$ atoms are responsible for the induced magnetic moments. Although the $p$ orbits of $\mathrm{O}$ atoms and $3 d$ orbits of $\mathrm{Zn}$ atoms also exhibit spin polarization, their contribution to the magnetic moment of the systems is small. Therefore, the origin of magnetism in $\mathrm{RE}$-doped $\mathrm{ZnO}$ monolayer resides on unpaired $f$ electrons of dopant $\mathrm{RE}$ atoms.

\section{Conclusions}

In summary, we have performed a comprehensive investigation of the structural, electronic, and magnetic properties of $\mathrm{ZnO}$ monolayer doped by $\mathrm{RE}(\mathrm{RE}=\mathrm{Y}, \mathrm{Ce}, \mathrm{Eu}, \mathrm{Gd}$, and Dy) using first-principles calculations. The doping of Ce, Eu, $\mathrm{Gd}$, and $\mathrm{Dy}$ in $\mathrm{ZnO}$ monolayers is found to be magnetic. $\mathrm{Y}$ doped $\mathrm{ZnO}$ monolayer exhibits no magnetism. The magnetic moment of RE-doped $\mathrm{ZnO}$ monolayer is mainly contributed from localized $f$ states of rare-earth atoms. Substitution doping of the RE atoms for $\mathrm{Zn}$ atom has significant effect on the electronic properties of $\mathrm{ZnO}$ monolayer. $\mathrm{ZnO}$ monolayer undergoes transition from semiconductor to metal in the presence of $\mathrm{Y}, \mathrm{Ce}, \mathrm{Gd}$, and Dy doping. More interestingly, Eu doped $\mathrm{ZnO}$ monolayer exhibits half-metallic behavior with a $100 \%$ spin polarization at the Fermi level. Our results may provide a reference for modifying the material property of $\mathrm{ZnO}$ monolayer and designing nanoelectronic and spintronic devices.

\section{Conflict of Interests}

The authors declare that there is no conflict of interests regarding the publication of this paper.

\section{Acknowledgments}

The authors acknowledge the support of National Natural Science Foundation of China (Grant nos. 51471064 and 51301054), the Program for New Century Excellent Talents (Grant no. 1253-NCET-009), and Program for Youth Academic Backbone in Heilongjiang Provincial University (Grant no. 1251G022).

\section{References}

[1] A. Janotti and C. G. Van de Walle, "Fundamentals of zinc oxide as a semiconductor," Reports on Progress in Physics, vol. 72, no. 12, Article ID 126501, 2009.

[2] S. J. Pearton, D. P. Norton, K. Ip, Y. W. Heo, and T. Steiner, "Recent progress in processing and properties of $\mathrm{ZnO}$," Progress in Materials Science, vol. 50, no. 3, pp. 293-340, 2005.

[3] Ü. Özgür, Y. I. Alivov, C. Liu et al., "A comprehensive review of $\mathrm{ZnO}$ materials and devices," Journal of Applied Physics, vol. 98, no. 4, Article ID 041301, 2005.

[4] A. Tsukazaki, A. Ohtomo, T. Onuma et al., "Repeated temperature modulation epitaxy for p-type doping and light-emitting diode based on ZnO," Nature Materials, vol. 4, no. 1, pp. 42-45, 2005.

[5] I. Repins, M. A. Contreras, B. Egaas et al., "19.9\%-efficient $\mathrm{ZnO} / \mathrm{CdS} / \mathrm{CuInGaSe}$, solar cell with $81.2 \%$ fill factor," Progress in Photovoltaics, vol. 16, no. 3, pp. 235-239, 2008.

[6] H. Morkoc and U. Ozgur, Zinc Oxide: Fundamentals, Materials and Device Technology, Wiley-VCH, Weinheim, Germany, 2009.

[7] C. Klingshirn, “ZnO: from basics towards applications," Physica Status Solidi B: Basic Research, vol. 244, no. 9, pp. 3027-3073, 2007.

[8] H. Guo, N. Lu, J. Dai, X. C. Zeng, X. Wu, and J. Yang, "Electronic structure engineering in chemically modified ultrathin $\mathrm{ZnO}$ nanofilms via a built-in heterointerface," RSC Advances, vol. 4, no. 36, pp. 18718-18723, 2014.

[9] H. Behera and G. Mukhopadhyay, "Strain-tunable band parameters of $\mathrm{ZnO}$ monolayer in graphene-like honeycomb structure," Physics Letters A, vol. 376, no. 45, pp. 3287-3289, 2012.

[10] M. H. Huang, S. Mao, H. Feick et al., "Room-temperature ultraviolet nanowire nanolasers," Science, vol. 292, no. 5523, pp. 1897-1899, 2001.

[11] H. Cao, J. Y. Xu, D. Z. Zhang et al., "Spatial confinement of laser light in active random media," Physical Review Letters, vol. 84, no. 24, pp. 5584-5587, 2000.

[12] Z. W. Pan and Z. L. Wang, "Nanobelts of semiconducting oxides," Science, vol. 291, no. 5510, pp. 1947-1949, 2001.

[13] S. Geburt, M. Lorke, A. L. da Rosa et al., "Intense intrashell luminescence of Eu-doped single $\mathrm{ZnO}$ nanowires at room temperature by implantation created $\mathrm{Eu}-\mathrm{O}_{i}$ complexes," Nano Letters, vol. 14, no. 8, pp. 4523-4528, 2014.

[14] M. Yin, Y. Gu, I. L. Kuskovsky et al., "Zinc oxide quantum rods," Journal of the American Chemical Society, vol. 126, no. 20, pp. 6206-6207, 2004.

[15] X. Y. Kong, Y. Ding, R. Yang, and Z. L. Wang, "Single-crystal nanorings formed by epitaxial self-coiling of polar nanobelts," Science, vol. 303, no. 5662, pp. 1348-1351, 2004.

[16] K. Govender, D. S. Boyle, P. B. Kenway, and P. O’Brien, "Understanding the factors that govern the deposition and morphology of thin films of $\mathrm{ZnO}$ from aqueous solution," 
Journal of Materials Chemistry, vol. 14, no. 16, pp. 2575-2591, 2004.

[17] K. T. Butler and A. Walsh, "Ultra-thin oxide films for band engineering: design principles and numerical experiments," Thin Solid Films, vol. 559, pp. 64-68, 2014.

[18] I. Demiroglu and S. T. Bromley, "Nanofilm versus bulk polymorphism in wurtzite materials," Physical Review Letters, vol. 110, no. 24, Article ID 245501, 2013.

[19] C. L. Freeman, F. Claeyssens, N. L. Allan, and J. H. Harding, "Graphitic nanofilms as precursors to eurtzite gilms: theory," Physical Review Letters, vol. 96, no. 6, Article ID 066102, 2006.

[20] F. Claeyssens, C. L. Freeman, N. L. Allan, Y. Sun, M. N. R. Ashfold, and J. H. Harding, "Graphitic nanofilms as precursors to wurtzite films: theory," Journal of Materials Chemistry, vol. 15, no. 1, pp. 139-148, 2005.

[21] C. Tusche, H. L. Meyerheim, and J. Kirschner, "Observation of depolarized $\mathrm{ZnO}(0001)$ monolayers: formation of unreconstructed planar sheets," Physical Review Letters, vol. 99, no. 2, Article ID 026102, 2007.

[22] G. Weirum, G. Barcaro, A. Fortunelli et al., "Growth and surface structure of zinc oxide layers on a $\operatorname{Pd}(111)$ surface," The Journal of Physical Chemistry C, vol. 114, no. 36, pp. 15432-15439, 2010.

[23] H. Y. Guo, Y. Zhao, N. Lu et al., "Tunable magnetism in a nonmetal-substituted $\mathrm{ZnO}$ monolayer: a first-principles study," The Journal of Physical Chemistry C, vol. 116, no. 20, pp. 11336$11342,2012$.

[24] A. L. He, X. Q. Wang, R. Q. Wu, Y. H. Lu, and Y. P. Feng, "Adsorption of an $\mathrm{Mn}$ atom on a $\mathrm{ZnO}$ sheet and nanotube: a density functional theory study," Journal of Physics Condensed Matter, vol. 22, no. 17, Article ID 175501, 2010.

[25] T. M. Schmidt, R. H. Miwa, and A. Fazzio, "Ferromagnetic coupling in a Co-doped graphenelike $\mathrm{ZnO}$ sheet," Physical Review B, vol. 81, no. 19, Article ID 195413, 2010.

[26] J. Ren, H. Zhang, and X. Cheng, "Electronic and magnetic properties of all 3d transition-metal-doped $\mathrm{ZnO}$ monolayers," International Journal of Quantum Chemistry, vol. 113, no. 19, pp. 2243-2250, 2013.

[27] F.-B. Zheng, C.-W. Zhang, P.-J. Wang, and H.-X. Luan, "Firstprinciples prediction of the electronic and magnetic properties of nitrogen-doped $\mathrm{ZnO}$ nanosheets," Solid State Communications, vol. 152, no. 14, pp. 1199-1202, 2012.

[28] K. Potzger, S. Zhou, F. Eichhorn et al., "Ferromagnetic Gdimplanted $\mathrm{ZnO}$ single crystals," Journal of Applied Physics, vol. 99, no. 6, Article ID 063906, 2006.

[29] X. J. Zhang, W. B. Mi, X. C. Wang, and H. L. Bai, "Firstprinciples prediction of electronic structure and magnetic ordering of rare-earth metals doped ZnO," Journal of Alloys and Compounds, vol. 617, pp. 828-833, 2014.

[30] Y. G. Zhang, G. B. Zhang, and Y. X. Wang, "First-principles study of the electronic structure and optical properties of Cedoped ZnO," Journal of Applied Physics, vol. 109, no. 6, Article ID 063510, 2011.

[31] H. Shi, P. Zhang, S.-S. Li, and J.-B. Xia, "Magnetic coupling properties of rare-earth metals ( $\mathrm{Gd}, \mathrm{Nd}$ ) doped $\mathrm{ZnO}$ : firstprinciples calculations," Journal of Applied Physics, vol. 106, no. 2, Article ID 023910, 2009.

[32] I. Bantounas, V. Singaravelu, I. S. Roqan, and U. Schwingenschlögl, "Structural and magnetic properties of Gd-doped ZnO," Journal of Materials Chemistry C, vol. 2, no. 48, pp. 1033110336, 2014.
[33] B. Delley, "An all-electron numerical method for solving the local density functional for polyatomic molecules," The Journal of Chemical Physics, vol. 92, no. 1, pp. 508-517, 1990.

[34] B. Delley, "From molecules to solids with the $\mathrm{DMol}^{3}$ approach," The Journal of Chemical Physics, vol. 113, no. 18, pp. 7756-7764, 2000.

[35] J. P. Perdew, K. Burke, and M. Ernzerhof, "Generalized gradient approximation made simple," Physical Review Letters, vol. 77, no. 18, pp. 3865-3868, 1996.

[36] Z. C. Tu and X. Hu, "Elasticity and piezoelectricity of zinc oxide crystals, single layers, and possible single-walled nanotubes," Physical Review B, vol. 74, no. 3, Article ID 035434, 2006.

[37] C. Freysoldt, B. Grabowski, T. Hickel et al., "First-principles calculations for point defects in solids," Reviews of Modern Physics, vol. 86, no. 1, pp. 253-305, 2014.

[38] J. Li, M. L. Hu, Z. Yu, J. X. Zhong, and L. Z. Sun, "Structural, electronic and magnetic properties of single transition-metal adsorbed BN sheet: a density functional study," Chemical Physics Letters, vol. 532, pp. 40-46, 2012.

[39] J. M. Zhang, D. Gao, and K. W. Xu, "The structural, electronic and magnetic properties of the $3 \mathrm{~d}$ TM (V, Cr, Mn, Fe, Co, Ni and $\mathrm{Cu}$ ) doped $\mathrm{ZnO}$ nanotubes: a first-principles study," Science China Physics, Mechanics and Astronomy, vol. 55, no. 3, pp. 428435, 2012.

[40] T. Minami, T. Yamamoto, and T. Miyata, "Highly transparent and conductive rare earth-doped $\mathrm{ZnO}$ thin films prepared by magnetron sputtering," Thin Solid Films, vol. 366, no. 1-2, pp. 63-68, 2000. 

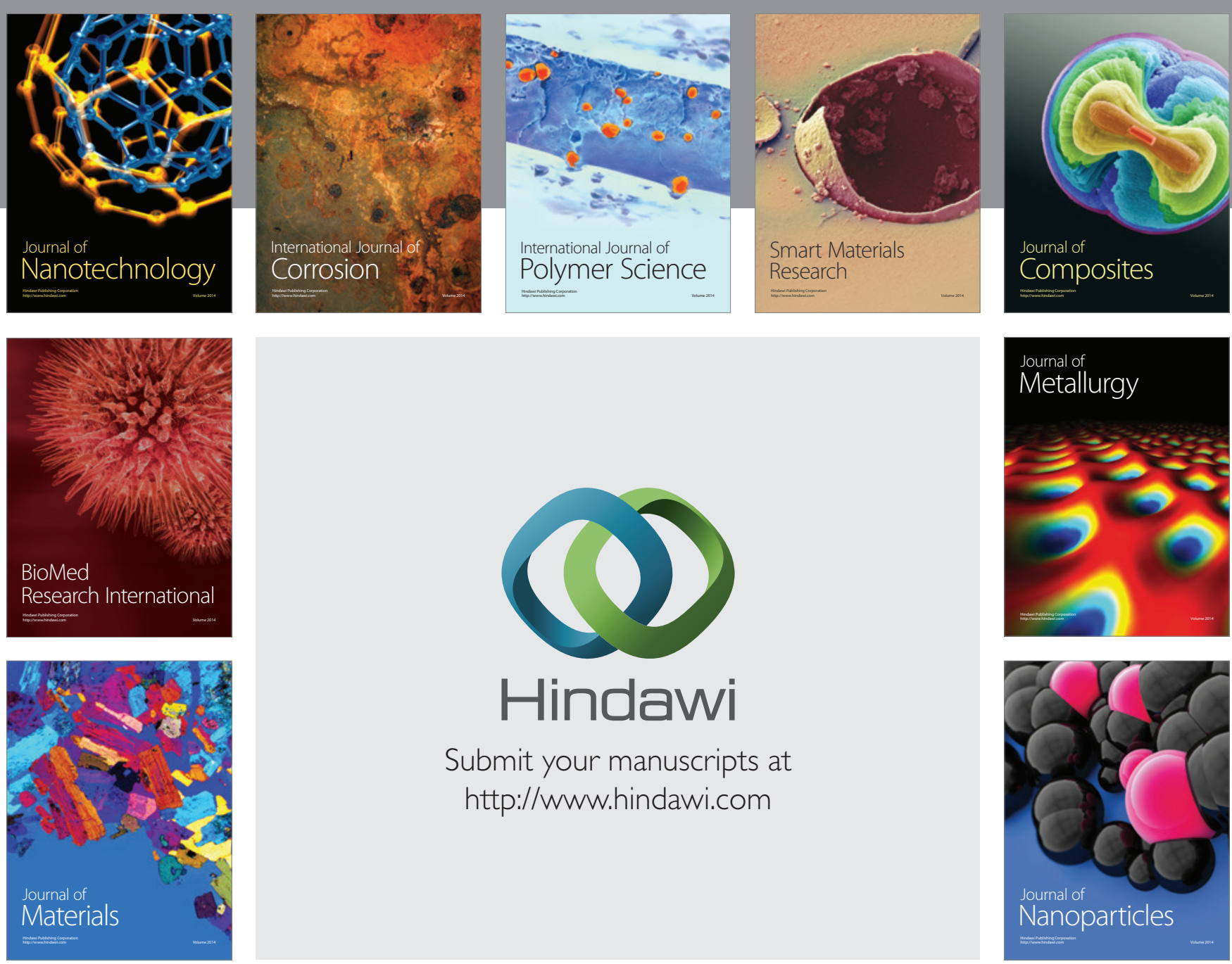

Submit your manuscripts at http://www.hindawi.com
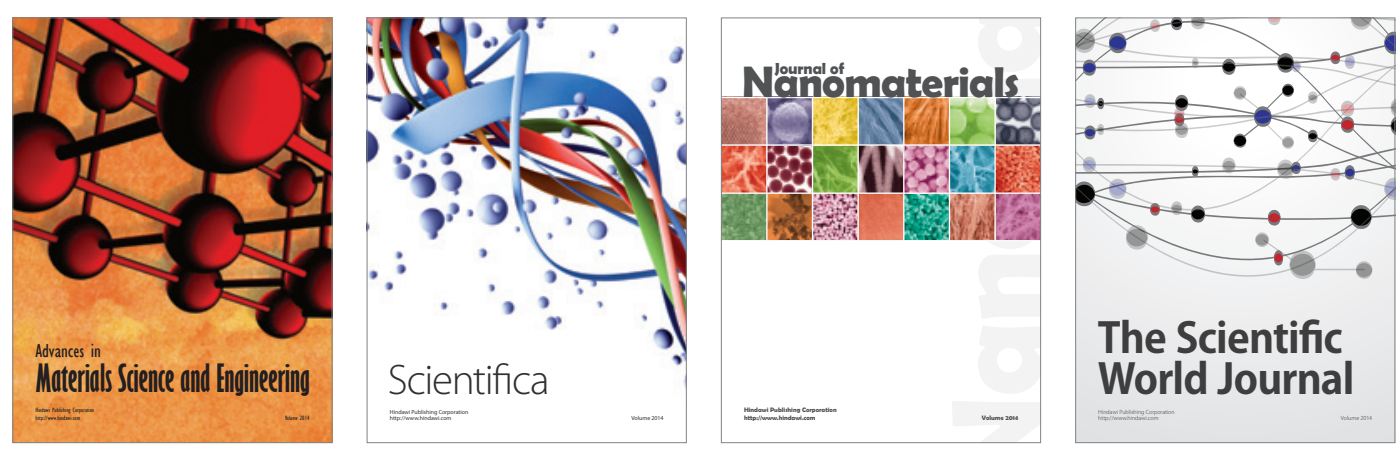

\section{The Scientific World Journal}
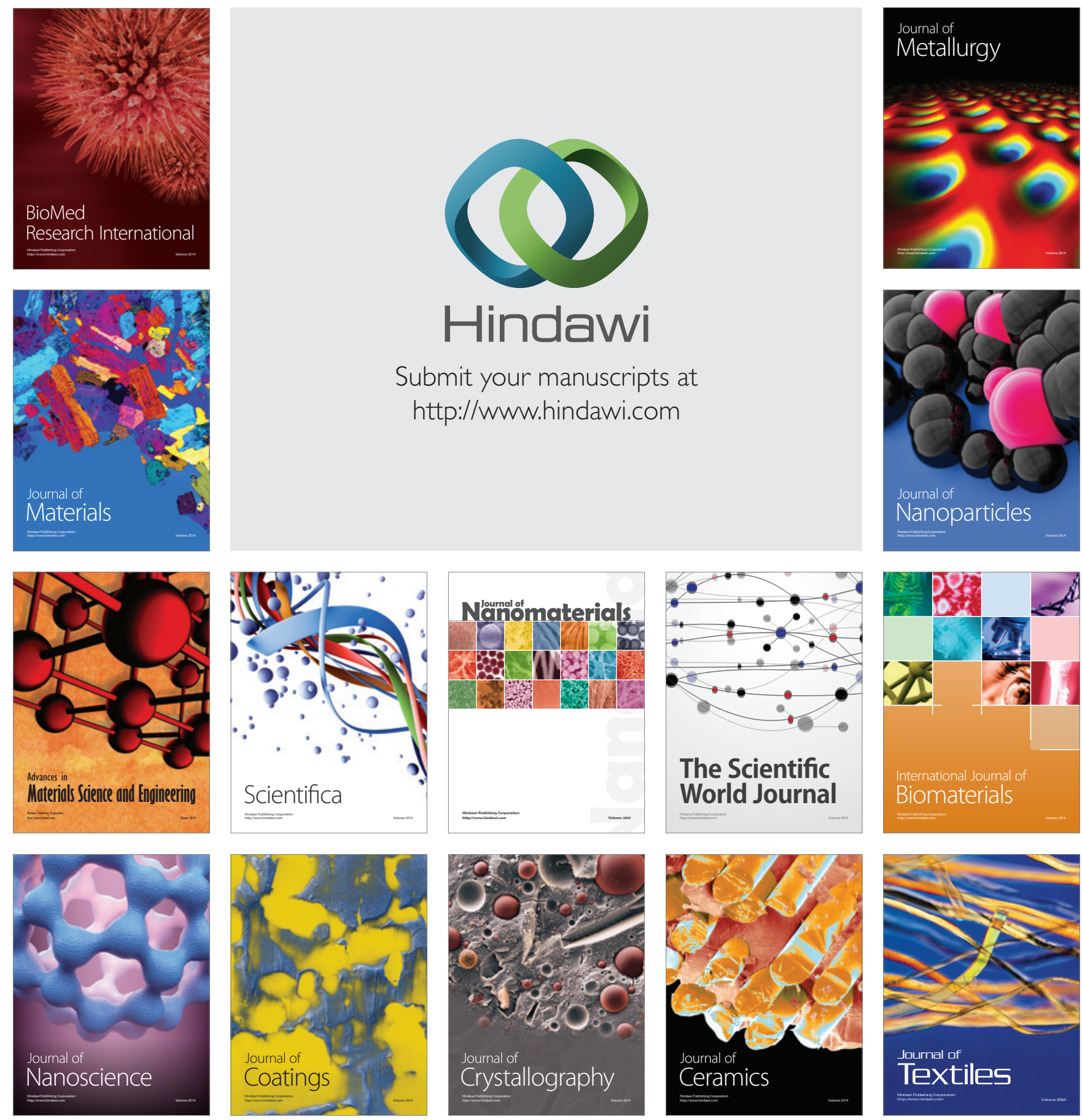